\title{
Construção de um modelo de evolução farmacêutica em prontuário médico
}

\author{
Construction of a model of pharmaceutical evolution \\ in patient's medical records
}

Recebido em: 21/12/2019 Aceito em: $07 / 06 / 2019$

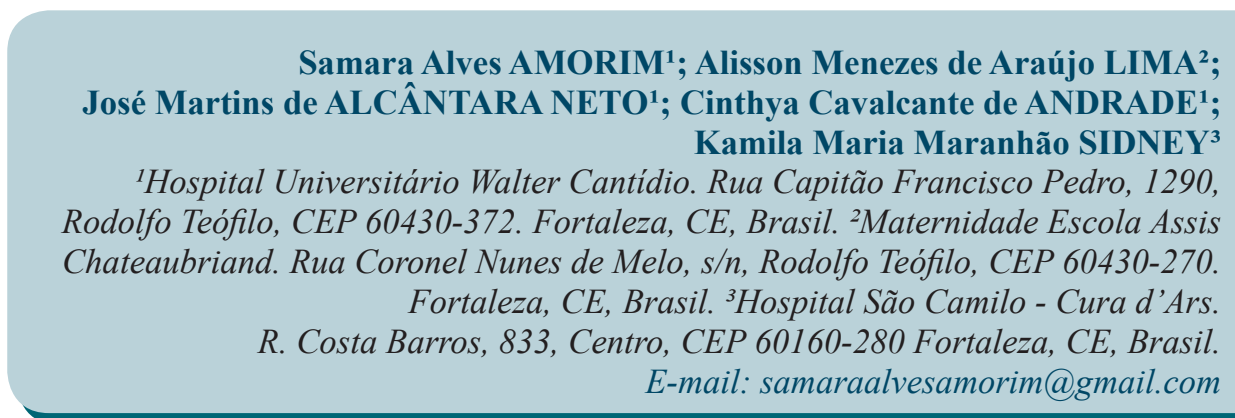

ABSTRACT

Considering the need to standardize the elaboration of the registry of the care provided to the patient by Health Workers, a model of pharmaceutical evolution record is proposed to be used by Clinical Pharmacists in a hospital scope. From a literature review of the narrative type, it began with the problem question:" what and how should the clinical pharmacist register in the patient's medical records at the hospital scope after the pharmaceutical anamnesis?", followed by for the search of articles in databases as Lilacs, Scielo and PubMed, and then a critical analysis and selection of papers that supported the construction of the proposed pharmaceutical evolution. Ten articles and 2 guidelines were selected, in which the organization of data was chosen from SOAP, which is an acronym in which each initial letter represents one of the sections of the notes of evolution, that is, subjective data (S), objective data (O), assessment (A) and planning (P). The data aimed to ensure that all patient pharmacotherapy is adequate to treat the involved health problem. Documenting the pharmaceutical care, in a standard form, led to the optimization of patient pharmacotherapy, and showed to be a necessary step to improve the communication in multi-professional teams. Therefore, the development of a pharmaceutical evolution SOAP can be useful to improve this point, by addressing what information and how to organize them.

Keywords: Pharmacist; hospital pharmacy service; Pharmaceutical Assistance; documentation.

\section{RESUMO}

Considerando a necessidade de padronizar a elaboração do registro dos cuidados prestados ao paciente, o presente trabalho propõe um modelo de nota de evolução farmacêutica para ser utilizada pelo farmacêutico clínico no âmbito hospitalar. Uma revisão de literatura do tipo narrativafoi iniciada com a questão-problema: 'o quê e como o farmacêutico clínico deve registrar, no prontuário do paciente em âmbito hospitalar, após realizar a anamnese farmacêutica?", seguida de pesquisa de artigos nas bases de dados Lilacs, Scielo e PubMed, e posteriormente, de uma análise crítica e da seleção de trabalhos para a construção da proposta de evolução farmacêutica. Foram selecionados 10 artigos e 2 guias. Foi feita a opção pela organização dos dados a partir do método SOAP, que é um acrônimo no qual cada letra representa uma das seções das 
anotações da evolução, ou seja, os dados subjetivos (S), os dados objetivos (O), a avaliação (A) e o planejamento $(\mathrm{P})$. Os dados abordados visaram garantir que toda a farmacoterapia do paciente esteja adequada para tratar os problemas de saúde. Documentar, de forma padronizada, os cuidados farmacêuticos prestados na otimização da farmacoterapia do paciente, é uma etapa necessária na melhoria da comunicação nas equipes multiprofissionais. Assim, a proposta de evolução farmacêutica pode ser útil, por abordar quais informações são relevantes e como organizá-las dentro do SOAP.

Palavras chaves: Farmacêutico; serviço de farmácia hospitalar; Assistência Farmacêutica; documentação.

\section{INTRODUÇÃO}

A integração do farmacêutico na equipe multiprofissional de assistência à saúde está se tornando cada vez mais comum e, assim, surgiu a necessidade de uma comunicação objetiva e eficaz com os demais profissionais. Dessa forma, é recomendada a padronização, em prontuário, dos registros dos serviços prestados (1).

Apesar da importância do registro em prontuário, muitos farmacêuticos não possuem essa experiência na prática. São descritas, como potenciais barreiras à documentação pelos farmacêuticos, a confiança excessiva na comunicação oral, receio de uma má aceitação pelos demais profissionais, além de outras implicações como a falta de tempo disponível e implementação incompleta ou inexistente de políticas institucionais de documentação dos serviços prestados ao paciente (2).

No Brasil, o Conselho Federal de Farmácia (CFF), em 2013, ao publicar a resolução $n^{\circ} 585$, destacou a importância do registro em prontuário pelo farmacêutico clínico, ao trazer o termo "evolução farmacêutica", e definindo o registro como uma das atribuições do farmacêutico. A Evolução Farmacêutica, assim, tem como finalidade documentar, no prontuário, o cuidado prestado ao paciente (3). Independentente do método clínico utilizado para a resolução de problemas, ter uma abordagem lógica e sistemática para o registro é parte fundamental no processo de assistência (4).

Os modelos de documentação clínica padronizados que podem ser adotados para a evolução farmacêutica incluem o SOAP, (subjective, objective, assessment, plan), TITRS (title, introduction, text, recommendation, signature) e FARM (finding, assessment, recommendations/resolutions, management), sendo o SOAP um modelo intervencionista e o mais difundido; o TITRS, um modelo de avaliação; e o FARM, uma ferramenta importante na monitorização (5).

Vista a necessidade de padronizar o registro dos cuidados prestados pelo farmacêutico clínico no prontuário do paciente no contexto hospitalar, o presente trabalho teve como objetivo propor um modelo de evolução farmacêutica.

\section{MÉTODO}

Trata-se de uma revisão de literatura do tipo narrativa. Assim, a partir da questão-problema: “o que e como o farmacêutico clínico deve registrar no prontuário do paciente em âmbito hospitalar após realizar a anamnese farmacêutica?" foi feita uma pesquisa na literature, durante o mês de julho de 2018, utilizando as bases de dados Lilacs, Scielo e PubMed. Na primeira pesquisa foram utilizadas as palavras-chave clinical pharmacist AND documentation AND health records. Na segunda pesquisa as palavras-chave utilizadas foram clinical pharmacist AND SOAP.

A segunda etapa foi a análise crítica dos artigos para a construção do modelo de evolução farmacêutica. Os critérios para inclusão dos artigos foram: abordagem do tema registro clínico e/ou registro pelo farmacêutico; ser escrito nos idiomas português, inglês ou espanhol; ter sido publicado a partir o ano de 2000. Não houve restrição para artigos que abordassem o tema registro clínico/evolução farmacêutica em outros contextos que não fossem o hospitalar, como por exemplo ambulatório ou farmácia comunitária

\section{RESULTADOS E DISCUSSÃO}

Como resultado das pesquisas nas bases de dados, foram encontrados, na primeira busca, 
138 artigos no Pubmed, 1 no Scielo; na segunda busca, foram encontrados 14 artigos no Pubmed. Foram selecionados 10 artigos e 2 Diretrizes (Quadro 1). Após a análise, foi proposto um modelo de evolução farmacêutica para registro em prontuário do paciente em âmbito hospitalar após anamnese farmacêutica pelo farmacêutico clínico (Quadro 2).

O prontuário é definido como documento único constituído por um conjunto de informações sobre a saúde do paciente e a assistência a ele prestada, que possibilita a comunicação entre os membros da equipe multiprofissional, a fim de contribuir na continuidade da assistência (6). Assim, ao considerar o farmacêutico integrado à equipe, dedicando-se à atenção ao paciente, suas atividades realizadas devem ser documentadas de forma sistemática e acessível (7).
Sob tal enfoque, o modelo de evolução farmacêutica, proposto neste trabalho (Quadro 2), visa contemplar a filosofia da prática da Farmácia Clínica, que é a provisão de serviços que visem garantir que toda a farmacoterapia do paciente seja adequada para tratar seus problemas de saúde, e que seja avaliada a sua efetividade, segurança e conveniência, além de trabalhar o uso adequado dos medicamentos (8).

A evolução farmacêutica faz parte do processo de cuidados farmacêuticos e consiste em coleta de dados objetivos e subjetivos, avaliação das informações, desenvolvimento de um plano de cuidado centrado no paciente, implementação do plano junto ao paciente e/ou aos demais profissionais de saúde e, por ultimo, follow-up, ou seja, monitoramento e avaliação da efetividade do plano. A documentação, acomunicação e a colaboração com a equipe são pontos centrais desse processo (9).

Quadro 1. Artigos selecionados, utilizando as palavras-chave clinical pharmacist AND documentation AND health records, clinical pharmacist AND SOAP (julho de 2018)

\begin{tabular}{|l|l|c|}
\hline Ano & \multicolumn{1}{|c|}{ Título do trabalho } & Referência \\
\hline 2017 & $\begin{array}{l}\text { Integration and assessment of the situation-background-assessment-recommendation framework into a } \\
\text { pharmacotherapy skills laboratory for interprofessional communication and documentation }\end{array}$ & $(1)$ \\
\hline 2017 & Assessment of SOAP note evaluation tools in colleges and schools of pharmacy & $(12)$ \\
\hline 2017 & $\begin{array}{l}\text { Development of a Documentation Rubric and Assessment of Pharmacists' Competency for } \\
\text { Documentation in the Patient Health Record }\end{array}$ & $(19)$ \\
\hline 2016 & Thinking Clinically from the Beginning: Early Introduction of the Pharmacists' Patient Care Process \\
\hline 2016 & Perceptions of pharmacy students and pharmacists on SOAP note education and in pharmacy practice & $(10)$ \\
\hline 2014 & Clinical Pharmacy Should Adopt a Consistent Process of Direct Patient Care. & $(21)$ \\
\hline 2014 & Standards of Practice for Clinical Pharmacists. Pharmacotherapy & $(14)$ \\
\hline 2013 & Documenting Clinical Activities & (20) \\
\hline 2010 & Pharmacists' documentation in patients' hospital health records: issues and educational implications & (2) \\
\hline 2007 & Clinical documentation for patient care: models, concepts, and liability considerations for pharmacists. & $(5)$ \\
\hline 2007 & Documenting Patient Care Services & $(11)$ \\
\hline 2003 & Guidelines on Documenting Pharmaceutical Care in Patient Medical Records & $(15)$ \\
\hline
\end{tabular}

O modelo de evolução proposto requer informações para as quais é necessário que o Farmacêutico Clínico, no processo de cuidado, realize a anamnese farmacêutica ( compreendida como procedimento de coleta de dados sobre o paciente), com a finalidade de conhecer sua história de saúde, elaborar o perfil farmacoterapêutico e identificar problemas relacionados $(4,10)$.

Para a organização dos dados do paciente, foi feita a opção, como escopo da evolução, pelo SOAP, que é um acrônimo no qual cada letra 1 re- presenta uma das seções das anotações da evolução diária, ou seja, os dados subjetivos (S), os dados objetivos $(\mathrm{O})$, a avaliação $(\mathrm{A})$ e o planejamento $(\mathrm{P})$ (11). Tem como pontos positivos ser o principal método de documentação utilizado por profissionais de saúde em ambientes hospitalares e ambulatoriais, não necessitar de um formulário específico e simplificar o registro (12).

Assim, o primeiro ponto do SOAP é o subjetivo, seção que fornece subsídio para avaliação e plano, e é nesse momento que o paciente é apre- 
sentado, com a descrição do diagnóstico e suas comorbidades (13). Na história da medicação, devem ser descritos a medicação do paciente e os problemas de saúde relacionados, e as informações sobre o uso e a adesão à terapia. Outro ponto a ser considerado é o registro de alergias relacionadas a medicamentos, pois pode afetar diretamente a prescrição, o monitoramento ou mesmo impedir o uso de determinados medicamentos (14).

No item objetivo, informações objetivas são aquelas clinicamente observáveis e normalmente incluem sinais e sintomas vitais e dados laboratoriais ou diagnósticos relevantes (12). Tanto a American Pharmacists Association (APhA) quanto a American Society of Health-System Pharmacists (ASHP) indicaram que essas informações sejam inseridas nesta seção, destacando também o registro de dados farmacocinéticos pertinentes à farmacoterapia $(11,15)$.

Com base nos dados subjetivos e objetivos, no tópico avaliação é esperada, do farmacêutico, a capacidade de identificar problemas relacionados aos medicamentos (PRM), ou seja, eventos ou circunstâncias envolvendo a farmacoterapia que, de fato ou potencialmente, interfiram nos objetivos pretendidos. Existem diferentes sistemas de classificação de PRM como, por exemplo, o Consenso de Granada (16).

A American Society of Health-System Pharmacists descreveu alguns PRM que podem ocorrer, e pontuou a importância de avaliar e registrar os ajustes feitos, na farmacoterapia, relacionados à posologia, adequação de via de administração, duplicidade terapêutica, identificação de interações entre medicamento-medicamento, medicamento-alimento, medicamento-exames laboratoriais, e, quando houver, registrar o aparecimento de reações adversas e sinais de toxicidade relacionados aos medicamentos (15).

Deve seja avaliado, pelo Farmacêutico Clínico, o uso adequado dos protocolos institucionais de profilaxias que envolvam medicamentos, como o protocolo de profilaxia de úlcera de estresse, no qual a avaliação, pelo farmacêutico, tem sido associada à diminuição do uso inapropriado de medicamentos por pacientes que não tinham ou deixaram de ter critérios para profilaxia medicamentosa, bem como à redução de custos associados ao uso desnecessário de medicamentos (17).

Quadro 2. Proposta de Modelo de Evolução Farmacêutica para Farmacêuticos Clínicos em âmbito hospitalar. 2018.

\begin{tabular}{|c|c|}
\hline Categoria & Informações para registro \\
\hline $\begin{array}{l}\text { Dados Subjetivos } \\
\text { e Objetivos }\end{array}$ & $\begin{array}{l}\text { - } \quad \text { Nome, sexo, idade. } \\
\text { - } \text { Motivo da Admissão. } \\
\text { - } \quad \text { História da medicação: problemas de saúde e medicamentos relacionados, adesão e alergias a } \\
\text { medicamentos. } \\
\text { - Lista atual de medicamentos. } \\
\text { - Sinais e sintomas relevantes para a farmacoterapia. } \\
\text { - } \quad \text { Dados de farmacocinética relevantes para farmacoterapia. } \\
\text { Parmetros laboratoriais. }\end{array}$ \\
\hline Avaliação & $\begin{array}{l}\text { - } \quad \text { Problemas Relacionados com Medicamentos (atuais e potenciais). } \\
\text { - } \quad \text { Adequação a protocolos institucionais de profilaxias que envolvam medicamentos. }\end{array}$ \\
\hline Plano & $\begin{array}{l}\text { - } \quad \text { justes da farmacoterapia. } \\
\text { - } \quad \text { Recomendações a serem feitas e/ou discutidas. } \\
\text { - } \quad \text { Regicação e/ou aconselhamento ao paciente ou a equipe relacionado a medicamento. } \\
\text { - } \quad \text { Parâmetros a serem monitorados. } \\
\text { - Data para reavaliação paciente. }\end{array}$ \\
\hline
\end{tabular}

A parte final do método SOAP é o plano de cuidados e condutas, onde devem ser descritas as informações relevantes para que as metas terapêuticas sejam cumpridas, bem como pontos para resolução que envolvam os PRM, e os parâmetros clínicos e laboratoriais que devem ser monitorados na determinação da necessidade, efetividade e segurança da farmacoterapia do paciente. Além disso, ações de educação em saúde para o uso correto de medicamentos e, se necessária, a data para rea- 
valiação $(18,19)$. Nesse ponto, também devem ser registradas as recomendações farmacêuticas (RF), visto que a RF é caracterizada como o ato planejado, documentado e realizado junto ao usuário e/aos profissionais de saúde, e que compõe o processo de acompanhamento farmacoterapêutico $(4,11)$.

Idealmente, deve ser usado um sistema eletrônico para registro; no entanto, caso o registro seja manuscrito, que seja de modo legível, utilizando caneta de tinta permanente, evitando rasuras e o uso de corretivos. Seja eletrônico ou manuscrito, deve ser carimbados e assinado. Deve ser evitado o uso de palavras de julgamento, opiniões sobre outros profissionais. $\mathrm{O}$ uso de abreviações deve ser limitado a apenas as já padronizadas pelo serviço (11). Ainda, é recomendado o uso, nos registros no prontuário, de termos como "sugerir" ou "considerar" em vez de "fazer" ou "precisar" (20).

Deve ser reforçada a importância do uso do registro, visto que a comunicação é uma habilidade essencial para todos os profissionais de saúde. Embora a comunicação oral possa ser o método preferido, em geral, a comunicação escrita pode resultar em melhores resultados para os pacientes (2). A nota de evolução deve ser feita de modo contínuo de forma a permitir a avaliação dos resultados e a identificação de quaisquer novas necessidades ou problemas relacionados à farmacoterapia (14).

Chan e cols (2016) pontuaram que, para padronizar o uso do SOAP, é necessário treinamento para a melhoria contínua da qualidade da documentação. Nos Estados Unidos da América, estudantes de farmácia são =ensinados a escrever notas SOAP no início da graduação, de modo que estejam familiarizados com o formato de nota e sejam capa- zes de incorporar a técnica em suas avaliações de pacientes, independentemente da complexidade do caso (21). Assim, o farmacêutico deve ser inserido no Procedimento Operacional Padrão da instituição, que indica quais profissionais estão autorizados a realizar a evolução no prontuário eletrônico do paciente, junto à Comissão de Prontuários (7).

As dificuldades encontradas na elaboração deste trabalho foram o número limitado de trabalhos nacionais, sobre o tema, na área da farmácia. Apesar do CFF, por meio das Resoluções n $n^{0}$ 555/2011 e $585 / 2013$, regular o registro do farmacêutico quando este preste seus serviços, não há regulamentação sobre o quê e como o farmacêutico deve realizar o registro em prontuário, como acontece em outras profissões de saúde como Enfermagem e Medicina (3, 22).

Como limitações do modelo proposto é necessário que a instituição tenha Farmacêuticos Clínicos em número proporcional ao número de pacientes avaliados; que o Farmacêutico esteja integrado à equipe assistencial; e que, idealmente, participe dos rounds clínicos, por ser um momento importante para estabelecimento de metas e objetivos para o paciente.

\section{CONCLUSÃO}

$\mathrm{O}$ ato de documentar, de forma padronizada, os cuidados farmacêuticos prestados na otimização da farmacoterapia do paciente, é uma etapa necessária na melhoria da comunicação nas equipes multiprofissionais. E, nesse sentido, o modelo de evolução farmacêutica proposto pode ser útil por abordar quais informações são necessárias e como organizá-las em SOAP.

\section{REFERÊNCIAS}

1. Barnett S, Nagy MW, Hakim RC. Integration and assessment of the situation-background-assessment-recommendation framework into a pharmacotherapy skills laboratory for interprofessional communication and documentation. Curr Pharm Teach Learn. 2017;9(5):794801. DOI: 10.1016/j.cptl.2017.05.023.

2. Pullinger W, Franklin BD. Pharmacists' documentation in patients' hospital health records: issues and educational implications. Int J Pharm Pract. 2010; 18(2):108-115. DOI: 10.1211/ijpp.18.02.0006.
3. CFF. Conselho Federal de Farmácia. Resolução no 585 , de 29 de agosto de 2013. Regulamenta as atribuições clínicas do farmacêutico e dá outras providências. Diário Oficial da União, 25 set 2013; Seção 1.

4. CFF. Conselho Federal de Farmácia. Serviços farmacêuticos diretamente destinados ao paciente, à família e à comunidade: contextualização e arcabouço conceitual. Brasília: Conselho Federal de Farmácia, 2016. 200 p. 
5. Zierler-Brown S, Brown TR, Chen D, Blackburn RW. Clinical documentation for patient care: models, concepts, and liability considerations for pharmacists. Am J Health Syst Pharm. 2007;64(17):1851-1858. DOI: 10.2146/ajhp060682.

6. CFM. Conselho Federal de Medicina. Resolução $\mathrm{n}^{\circ}$ 1.638 , de 9 de agosto de 2002. Define prontuário médico e torna obrigatória a criação da Comissão de Revisão de Prontuários nas instituições de saúde. Diário Oficial da União, 10 jun 2002; Seção 1. p. 184-5.

7. Lima ED, Silva RG, Ricieri MC, Blatt CR. Farmácia clínica em ambiente hospitalar: enfoque no registro das atividades. Rev. Bras. Farm. Hosp. Serv. Saúde. 2017;8(4):18-24. DOI: 10.30968.

8. Cipolle RJ, Strand LM, Morley PC. Pharmaceutical Care Practice: the patient centered to medication management. 3rd ed. New York: McGraw-Hill, 2012. 697p.

9. JCPP. Joint Commission of Pharmacy Practitioners. Pharmacists' Patient Care Process. 2014. Disponível em: https://jcpp.net/wp-content/uploads/2016/03/PatientCareProcess-with-supporting-organizations.pdf.

10. Thinking Clinically from the Beginning: Early Introduction of the Pharmacists' Patient Care Process. Am J Pharm Educ. 2016;80(10):164. DOI: 10.5688/ajpe8010164.

11. Buffington DE, Bennett MS. Medication therapy management services. Documenting patient Care Services. Mod. 5. American Pharmacists Association. 2007.

12. Sando KR, Skoy E, Bradley C, Frenzel J, Kirwin J, Urteaga E. Assessment of SOAP note evaluation tools in colleges and schools of pharmacy. Curr Pharm Teach Learn. 2017; 9(4):576-584. DOI: 10.1016/j.cptl.2017.03.010.

13. Lew V, Ghassemzadeh S. SOAP note [on-line]. Treasure Island: StatPearls Publishing; 2018. Available at: www. ncbi.nlm.nih.gov/books/NBK482263/.

14. ACCP. American College of Clinical Pharmacy. Standards of Practice for Clinical Pharmacists. Pharmacother. 2014;34(8):794-797.
15. ASHP. American Society of Health-System Pharmacists. Guidelines on documenting pharmaceutical care in patient medical records. Am J Health-Syst Pharm. 2003;60:705-707.

16. Adusumilli PK, Adepu R. Drug related problems: an over view of various classification systems. Asian J Pharm Clin Res. 2014;7(4):7-10.

17. Buckley MS, Park AS, Anderson CS, Barletta JF, Bikin DS, Gerkin RD, O’Malley CW, Wicks LM, Garcia-Orr R, Kane-Gill SL. Impact of a clinical pharmacist stress ulcer prophylaxis management program on inappropriate use in hospitalized patients. Am J Med. 2015;128:905-913. DOI: 10.1016/j.amjmed.2015.02.014.

18. Harris IM, Phillips B, Boyce E, Griesbach S, Hope C, Sanoski C, Sokos D, Wargo K. Clinical Pharmacy Should Adopt a Consistent Process of Direct Patient Care. Pharmacother. 2014; 34(8):133-148. DOI: 10.1002/ phar.1459.

19. Baranski B, Bolt J, Albers L, Siddiqui R, Bell A, Semchuk W. Development of a Documentation Rubric and Assessment of Pharmacists' Competency for Documentation in the Patient Health Record. Can J Hosp Pharm. 2017;70(6):423-429.

20. SHPA. Society of Hospital Pharmacists of Australia. Documenting Clinical Activities. J Pharm Pract Res. 2013; 43(2): 42-46.

21. Chan A, Saeteaw M, Chui WK, Lee JY. Perceptions of pharmacy students and pharmacists on SOAP note education and in pharmacy practice. Curr Pharm Teach Learn. 2016;8(1):77-82. DOI: 10.1016/j.cptl.2015.10.001.

22. CFF. Conselho Federal de Farmácia. Resolução n ${ }^{0} 555$, de 30 de novembro de 2011. Regulamenta o registro, a guarda e o manuseio de informações resultantes da prática da assistência farmacêutica nos serviços de saúde. Diário Oficial da União, 14 dez 2011; Seção 1. 